\title{
Multi-Agent Radio Resource Allocation
}

\author{
Clemens Kloeck • Holger Jaekel • Friedrich Jondral
}

(C) Springer Science + Business Media, LLC 2006

\begin{abstract}
This paper presents a spatially distributed and dynamic billing, pricing and allocation mechanism for which a user terminal requires Cognitive Radio abilities. That is, the Cognitive Radio abilities will be applied to the economical environment. The radio resource goods are allocated to the users by a multi-unit sealed-bid auction. Intelligent entities like the bidding strategy have to represent operator's and users' behavior and to make decisions for them in order to fulfill the preferences and QoS. The main functionalities, used to execute the dynamic auction sequence and located in the MAC are described, assuming the entities possess Cognitive Radio abilities.
\end{abstract}

Keywords auction $\cdot$ cognitive radio $\cdot$ multi agent systems $\cdot$ radio resource management

\section{Introduction}

In communication systems the radio functionalities tend toward more and more intelligent algorithms. Their ability to react on different influences in an appropriate and sophisticated manner will increase. In

C. Kloeck $(\bowtie) \cdot$ H. Jaekel · F. Jondral

Communication Labs, Universitaet Karlsruhe (TH),

76133 Karlsruhe, Germany

e-mail: kloeck@int.uni-karlsruhe.de

H. Jaekel

e-mail: jaekel@int.uni-karlsruhe.de

F. Jondral

e-mail: fj@int.uni-karlsruhe.de future, these radios will recognize and observe their environment and learn about it. The learning process results in modified actions and adaption to the environment. This cognition and the following execution can be realized in a Cognitive Radio [1]. One of the first steps towards a cognitive radio will be introduced in this paper by simultaneously allocating spectrum and determining the price per bandwidth in periodically repeated auctions.

In established billing systems the prices are fixed and the customers who demand first will be served first. Auctions allow the customers to incorporate their needs and demands instantaneously into the good allocation at the current market situation. Therefore, the price depends on the willingness of a customer to pay for goods currently offered. Clearly, the auctioneer has the possibility to influence the market situation by announcing a reserved price. Each bid has to exceed this limitation in order to be accepted to the auction. Both sides, thus, have the opportunity to influence the market progress in real time.

Medium access control mechanisms can be divided in deterministic and statistical. One well-known representation of the last mechanism is ALOHA and its modified kind slotted-ALOHA [2]. On the other hand, several deterministic access mechanisms are proposed in order to avoid access interference. Besides the CSMA/CD [3] and CSMA/CA [4], mechanisms were proposed based on periodically repeated auctions. The so-called Resource Auction Multiple Access (RAMA) [5-7] is based on sequential sealed-bid singleunit auction which can be improved by using a multiunit sealed-bid auction. In contrast to RAMA for which the bids have no intention because of being randomly chosen, D-RAMA [8] allows to weigh the bids based 
on the buffer size of the proper service class. The aim is to reach a better quality of service. These protocols do not allow users to incorporate their purchase power, the experience of the past and additional information of the future.

The access and assignment mechanism proposed in this paper, allows users to express their needs, urgency to send, preferences, purchase power combined with a QoS-aware buffer management which categorized the data according to their urgency to send in order to fulfill the QoS criteria. The auction mechanism will be applied to offer Radio Resource Goods (RRGs) to users and allocate it after the auction. A multi-unit auction [9] assigns these spectrum parts to users according to their bids and at the same time determines the price depending on the bids and the auctioneer's reserved price. Hence, the auction mechanism incorporates both spectrum allocation and billing.

Since the repetition duration should be very small like seconds or even milliseconds, signaling effort represents a very important constraint concerning the type of auctions used for the resource allocation in wireless communication systems. Auction types like open auctions and sequential auctions [9] are not suitable, because the undetermined number of iterations leads to an unpredictable auction duration. On the other hand, the sealed-bid auctions are very fast. The auction duration is only a linear function of the number of bidders. The signaling commands are short and can be piggybacked into some free space of the header or the data packets. Therefore, the sealed-bid auction can be implemented under heavy time and signaling constraints.

This paper will be organized as follows: Section 2 gives an overview of the MAC using auctions. The proposed system and especially the bidding strategy, reserve price calculation and the auction mechanism are described in Sections 3-6. Finally, simulation results will illustrate the behavior of this system in Section 7.

\section{Auctions as MAC entities}

In communication systems, the operator represents the auctioneer's side and the users are the bidders. The Economic Manager (EM) responsible for the auction is logically located in the Medium Access Control layer (MAC) of a Radio Access Technology (RAT) in each cell and controls the bandwidth allocation by auctions. Its counterpart is located in the MAC-layer of the user terminal. The Radio Resource Goods (RRGs) offered which can be bandwidths $\Delta f$ leased for a certain time duration $\Delta T$. Every duration $\Delta T$ an auction takes place in which the free RRGs are offered. In this auction both the currently served users and the new yet unserved users participate.

In such a highly dynamical process the user cannot bid by himself. An RRG Auction Agent (RAA) located in the MAC-layer should represent the bidder's behavior. This behavior has to be expressed in a form, which an algorithm can handle. Therefore, users wishes, demands and behaviors should be represented by a set of parameters or functions, see Section 4.3. Not only private information can influence the bidding strategy, but also the experience of past auctions. Since a user participates in several auctions, the information of past allocation and price development may be the input to a prediction algorithm in order to improve the bidding strategy, see Section 4.

On the operator's side an auctioneer's agent located in the proper MAC-layer represents the operator's behavior. Its tasks include the announcement of an auction every $\Delta T$ time units, calculating and predicting the reserved price based on fixed costs and executing the auction mechanism.

EM and RAA act and react in a periodic auction sequence. In the past the focus in auction theory was mainly on auctions which occur once. This spectrum allocation approach needs a more sophisticated consideration of auctions. The auction sequence has to be considered as a dynamic process. The intention of maximizing expectations can be directly applied in this process, because a user takes part as often as his gain and his good allocation can be approximated by the expectations. Therefore, optimizing the bidding strategy with respect to the expectations makes sense.

From the economical point of view, this auction process is more suitable than established billing strategies concerning the operator's revenue in a frequently visited cell.

\section{System description}

\subsection{Overview}

A resource allocation method based on auctions will take place beyond $3 \mathrm{G}$. The auction is located in the EM and will be executed by the subentity Auction Mechanism (AM), see Fig. 1. The EM is logical located next to the Base Station (BS) within the MAC and is responsible for the RRGs allocation under economical aspects. The RRGs will be auctioned and the user who wins RRGs will get General Resource Elementary Credits (GRECs). Besides the EM at the operator's side, the RAA at the users' side comprises the three 


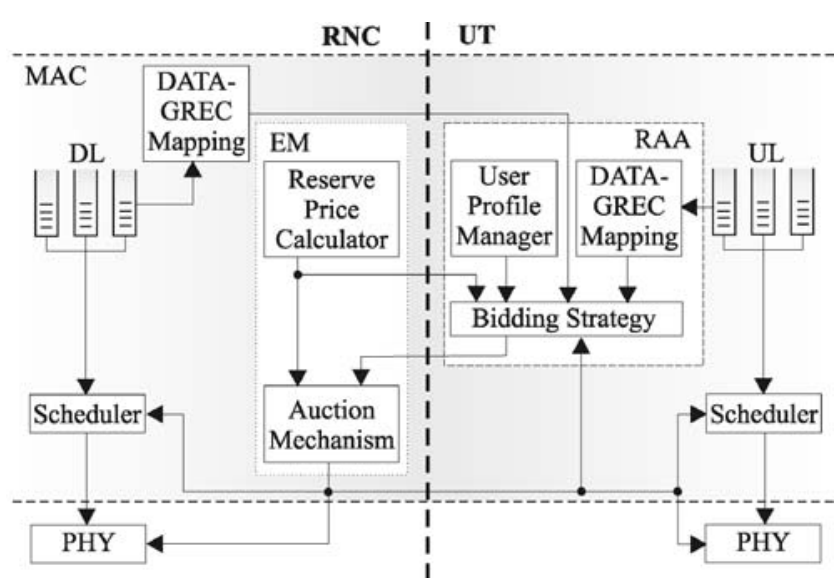

Figure 1 System model

main entities DATA-GREC-Mapping (DGM), Bidding Strategy (BIS) and User Profile Manager (UPM) which handles the bidding process. Both in the UpLink (UL) and the DownLink (DL) the entity DGM maps the MAC data of the QoS-classes and their parameters into GREC categories. These categories can mainly be distinguished between critical data which should be urgently sent within this auction period in order to meet the QoS and uncritical data which could be transmitted. This information are input to the entity BIS which is located in the User Terminal (UT). The BIS also needs user specific information about his preferences and purchase power provided by the entity UPM. Furthermore, BIS gets additional information to be executed the auction like the reserve price $r$ from the Reserve Price Calculator (RPC) and may predict the auction process like the behavior of the other bidders. The last information will provided by the allocation vector which is broadcasted to all participants.

This allocation method has both an operator's and a user's leverage. The RPC aims at maximizing the operator's monetary gain, whereas the RAA wants to satisfy the user's utility by minimizing costs. The auction mechanism has to be common knowledge to calculate both the reserve price $r$ and the bid vector bid $_{k}$. Section 3.2 describes the auction schedule. Sections 4-6 explain the functionalities of the BIS, the RPC and the AM, respectively.

\subsection{Auction schedule}

At first, the RPC calculates the reserve price $r$. The EM announces the auction at time $n T$ by broadcasting the number of GRECs $N_{\max }$ to be offered and the reserve price $r$ each bid has to exceed. The RAA receives this announcement and calculates the bid vector bid $_{k}$ based on the aforementioned information and on the knowl- edge of previous auctions. The EM collects all bid of the users who want to participate and allocates the GRECs according to the specific auction mechanism. The result will be transmitted by the allocation vector to each UT which can gain the information about the GRECs won and instruct the MAC scheduler and the PHY to send the data accordingly. Contemporarily, the information especially for the DL will be conveyed to the entities at the DL-side which are responsible for transmitting the data. After the data transmission, another auction starts at time $(n+1) T$.

\subsubsection{Signaling quantization}

Clearly real values and their corresponding floating point format go beyond the scope of a reduced signaling approach. An encoding protocol has to be defined to reduce the needed bits per message. The reserve price as well as the bids can be transmitted in a differential or absolute form.

In this paper the overall concept is considered. As the basestation admits the user, the same message will contain the absolute value of the minimum and maximum reserve price $r_{\min }, r_{\max }$ and the number of quantization steps $N_{b i t}$ of the interval $\left[r_{\min }, r_{\max }\right]$. In the following auction announcement only the quantized reserve price $r_{q}$ need to be broadcasted. The reserve price is the leverage of the operator to influence the market, resulting in an approximately similar variation of the reserve price $r_{q}$. The limits $r_{\text {min }}, r_{\text {max }}$ may change if most of the bids take the value of the maximum or minimum reserve prices. There are two reasons to change the two limits: first, the bids can be distinguished in a better manner leading to higher overall utility and second the price can be better adapted to the market resulting in a higher operator's gain. The EM transmits these borders during the auction seldomly, therefore the system can cope with an integer or even double value transmission.

The bids are quantized and the interval of the absolute bid has to be defined (see Fig. 2). The absolute bid has to be between the reserve price $r$ and the maximum reserve price $r_{\max }$. A bid is represented by $N_{b i t}$ bits. They are equidistantly spaced through $\left[r, r_{\max }\right]$, e.g., with $N_{b i t}=4$, the bid which is equal to the reserve price is represented by "0000," whereas the bid $b=r_{\max }$ is mapped to "1111." The higher the reserve price is, the more the bid quantization steps shrink. That is reasonable to better distinguish the bids in a shorter and simultaneously relatively higher interval. Based on the given maximum costs, a bid has not to be exceeded, the bid region for high bids has to be fine enough to be able to approximate as good as possible 
Figure 2 Quantization and storage of the histogram $h$, bid values $b$ and reserve price $r$

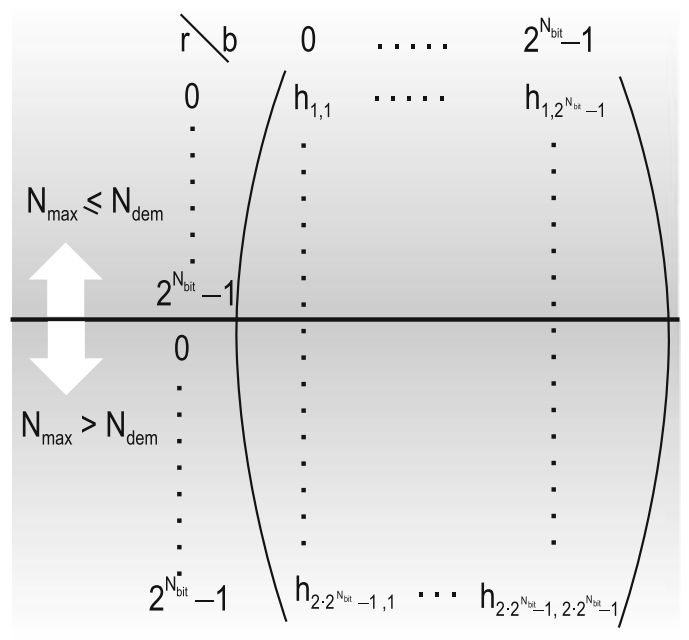

the maximum costs, resulting besides the fairness in a higher operator's gain.

\section{Bidding strategy}

The bidding strategy possesses an integral and a differential part. The integral part incorporates past auctions and thus the past users' behaviors in order to predict his number of GRECs won $N_{l}^{w, x, y}$ for QoS-buffer $l$ of link $y \in\{U L, D L\}$ and data category $x \in\{c r i$, uncri $\}$ depending on the proposed bidding vector bid. On the other hand, the differential part changes the bid value $b$ afterwards if the auction conditions like reserve price $r$ and number of demand $N_{d e m}$ have been kept stable, but the goals are not fulfilled.

\subsection{Strategy policy}

The design of the bidding strategy aims at satisfying the user's wishes, that is this algorithm tries to act as similar as the user would do. The actions goals can be sorted according to the following decreased-ordered priority list:

1) Keeping the budget constraint

2) Fulfillment of the QoS

3) Maximization of the utility

4) Minimization of the costs

As in normal live the bidding strategy will get a budget the costs must not exceed. The user adjusts this budget constraint with the UPM. This item is the most important restriction to the bidding strategy to keep the confidence to the user and to be allowed to act instead the users without permanent supervision. The budget constraint can be differential or cumulative. A differential budget constraint means that the maximum costs per QoS class, data category and link are given for one auction. On the other hand, a cumulative budget constraint summarizes all other opportunities like cumulative costs per auction, whereas the sum of the bid per auction must not exceed this limit. Additionally, the accumulation over time can be envisaged, that is cumulative costs over several auctions have to be below a proper threshold.

Within this economical framework the requested service should be executed as good as possible. A service is defined by a set of parameters. These parameters are determined fixed or tolerated within an interval to execute the service satisfactorily. In this case the second layer offers the third layer several different services. The convergence layer inbetween has to assign the incoming data to the proper Service Classes (SC). The data in a service classes can be categorized according to the urgency to send them with respect to the parameter specification fulfillment. If some or even all parameter specifications are injured the quality of service (QoS) suffers. Summarizing, the bidding strategy is responsible for awarding as much RRGs as necessary to transmit the data according to their urgency to fulfill the QoS. The bidding strategy will thus at first bid for the critical data and afterwards for the uncritical.

After this Preselection of the bids which optimally fulfill the QoS, the utility of transmitting the proper data will be determined und maximized. In other words, these data within each category but over the service classes will be selected for transmission which maximize the QoS. For this data the bidding strategy will submit bids bid.

Rationally, keeping in mind the leeway of the budget constraint for the bids in order to reach the goals of 1 ) 
and 2), the bidding strategy must be anxious to minimize the costs in line with the common users wishes.

\subsection{Input}

The bidding strategy gets its input from the QoS Buffer Management (QoS-BM) and the UPM. The QoS-BM determines the critical data $D^{c r i}$ which should be urgently sent and the correspondingly uncritical data $D^{u c r i}$ within this auction period $T$ and maps these data to the number of GRECs $N_{l}^{w, x, y}$ which are the goods to be auctioned per link and QoS and data category.

The UPM provides the bidding strategy with the user relevant data concerning his purchase power and preferences. The user can adjust for each QoS class in uplink/downlink a maximum value $\mathbf{c}_{l}^{x, y}$ that the cost per GREC must not to exceed. Thus, he is able to influence the price of the data of different QoS classes mainly representing different applications like realtime audio data for a phone call or best effort data for an email download.

Second, the user can express his preferences by a preference vector $\alpha$. Its components $\alpha_{l}^{x, y}$ indicate the relative personal evaluation of the QoS classes, e.g., if a user is more sensitive to a noisy phone call than to a disturbed FTP download, the QoS class mainly containing phone data will get an higher $\alpha_{l}^{x, y}$ than the one representing FTP.

The input to the BIS is represented as a set of quintuples $I$ :

$$
\begin{aligned}
I & =\left\{\left(\alpha_{l}^{x, y}, N_{l}^{b, x, y}, l, x, y\right) \mid l\right. \\
& =1 \ldots L, x \in\{c r i, u c r i\}, y \in\{u l, d l\}\}
\end{aligned}
$$

in which $L$ is the number of QoS classes of only one link. The vector $\mathbf{n}$ which components are elements of $I$ possesses the property:

$$
\begin{aligned}
\mathbf{n}_{i} & =\left(\alpha_{l, i}^{x, y}, N_{l, i}^{b, x, y}, l_{i}, x_{i}, y_{i}\right) \wedge \mathbf{n}_{j}=\left(\alpha_{l, j}^{x, y}, N_{l, j}^{b, x, y}, l_{j}, x_{j}, y_{j}\right) \\
& \left.\Rightarrow(i<j) \Leftrightarrow \alpha_{l, i}^{x, y}>, \alpha_{l, j}^{x, y}\right)
\end{aligned}
$$

i.e., the components are sorted in a decreasing order with respect to the preference $\alpha_{l}^{x, y}$.

\subsection{Utility function}

The bidding strategy aims at satisfying the user's utility function as good as possible and contemporarily saving money. The utility function $\eta_{l}^{x, y}\left(\alpha_{l}^{x, y}, N_{l}^{b, x, y}, R\right)$ com- bines the use of resources expressed by the number of GRECs $N_{l}^{b, x, y}$ needed to satisfy the QoS requirements of each class in uplink/downlink and the user preferences represented by a preference vector $\alpha$. The variable $R$ serves as a limiter of the utility. That is, the utility of $N_{l}^{b, x, y}>R$ remains the same regardless of any additional goods:

$$
\eta\left(\alpha_{l}^{x, y}, N_{l}^{b, x, y}, R\right)= \begin{cases}\eta\left(\alpha_{l}^{x, y}, R, R\right) & N_{l}^{b, x, y}>R \\ \eta\left(\alpha_{l}^{x, y}, N_{l}^{b, x, y}, R\right) & N_{l}^{b, x, y}<R\end{cases}
$$

The limitation property can be switched off by converging $R$ to infinity:

$$
\eta\left(\alpha_{l}^{x, y}, N_{l}^{b, x, y}\right)=\lim _{R \rightarrow \infty} \eta\left(\alpha_{l}^{x, y}, N_{l}^{b, x, y}, R\right) .
$$

The user can also choose his basic utility behavior for each QoS-class categorized by the well-known economic expressions: risk-averse, risk-neutral and riskencouraged.

If the utility function $\eta_{l}^{x, y}\left(., N_{l}^{b, x, y}, R\right)$ is risk averse in $N_{l}^{b, x, y}$, the differential utility $d \eta_{l}^{x, y}$ and in this sense the additional utility of more goods will decrease if $N_{l}^{b, x, y}$ increases. Considering a risk-neutral function, the differential utility $d \eta_{l}^{x, y}$ is equal for each additional differential good $d N_{l}^{b, x, y}$. If there exits a QoS class for which the QoS is only fulfilled sending complete datagrams, the user will choose a risk-encouraged function whose differential utility increases in $N_{l}^{b, x, y}$. All the utility functions have the following two properties in common:

1) $\eta_{l}^{x, y}\left(\alpha_{l}^{x, y}, 0, R\right)=0$

2) $\eta\left(\alpha_{1}, n_{0}, R\right)>\eta\left(\alpha_{2}, n_{0}, R\right) \Leftrightarrow \alpha_{1}>\alpha_{2}$

The first expression means there is no utility if no goods are available. The second item states the increased utility by an underlying higher preference assuming utility functions of the same category. Especially, for the risk neutral class another property holds with respect to the derivative $\eta^{\prime}$ :

3) $\eta^{\prime}\left(\alpha_{1}, \cdot, R\right)>\eta^{\prime}\left(\alpha_{2}, \cdot, R\right) \Leftrightarrow \alpha_{1}>\alpha_{2}$

This property mainly reduces the computer effort of finding the optimized bid vector.

\subsection{Risk-averse utility}

The differential utility of the critical data is constant in the most cases, because a lost critical datum is indistinguishable of its buffer location and temporal position. Consequently, the respective utility function 
is linear. The critical data of a QoS buffer does have to produce a higher utility than the uncritical. The importance and the additional characteristic of the critical data, e.g., to transmit, is apparent in comparison to the uncritical data. All the other characteristics are the same. The data in the QoS buffer can always be sorted to get a concave utility graph, if the order relation is the urgency to keep the QoS parameter limits and the differential utility is monotonically decreasing in the urgency. The data will be sorted in a descending order. Thus, the first data are the critical ones, followed by the uncritical. Last but not least to save computation power, this concave function can be approximated by two linear functions, a linear utility for each the critical and uncritical data. This is an approximation of the well-known risk-averse behavior observed in economic studies. The critical as well as the uncritical utility function $\eta\left(\alpha_{l}^{c r i, y}, N_{l}^{c r i, y}, L_{l}^{c r i, y}\right)$ and $\eta\left(\alpha_{l}^{u c r i, y}, N_{l}^{u c r i, y}, L_{l}^{u c r i, y}\right)$ can be described by the preference-limiter tuple $\left(\alpha_{l}^{x, y}, L_{l}^{x, y}\right)$.

\subsection{Utility criterion}

The main goal of the bidding strategy is to approach the utility function of the GRECs needed and the GRECs won. Therefore, the criterion of the difference function $\Delta_{\eta}(x, y)$ is defined as:

$\Delta_{\eta}(\mathbf{x}, \mathbf{L})=\sum_{i}\left(\eta_{l}^{x, y}\left(\alpha_{l, i}^{x, y}, x_{i}, L_{i}\right)-\eta_{l}^{x, y}\left(\alpha_{l, i}^{x, y}, L_{i}, L_{i}\right)\right)$.

The difference utility function is always non-positive and the value indicates the utility missed. The quadratic error utility is not chosen, because based on its minimization result, you cannot conclude to the utility maximization result. For example, considering two different $\mathbf{x}_{1}$ and $\mathbf{x}_{2}$, whereas both of them affect the same quadratic utility error, but the gained utility is different. Thus the $\mathbf{x}$ has to be chosen with the highest utility to reach utility maximization and not the one with the minimum quadratic utility error. Summarizing, minimizing the quadratic error utility there is no incentive to favor utility functions to get very close to their utility wanted, but gain less additional utility, over utility functions for which the difference to the utility wanted is higher, but gain more additional utility. Thus, the absolute utility maximization suffers from the quadratic error functions.

\subsection{Bid representation}

The bid vector bid $_{k}$ of user $k$ is a vector consisting of quadruple elements:

bid $_{k}=\times_{i}\left(N_{l, i}^{b, x, y}, b_{i}, y, l\right)$.

The information of one quadruple includes the number of RRGs $N_{l, i}^{b, x, y}$ needed for link $y$ and SC $l$. For each RRG of $N_{l, i}^{b, x, y}$ the RAA bids $b_{i}$. Depending on the RAT, DGM and auction protocol, the bid vector sized can be limited and information can be rejected.

\subsection{Ideal strategy}

Ideally, the bidding strategy possesses complete information of the other users inter alia their bids. The goods a bidder wins is a deterministic function of his own bids bid and the bids of the other users bid $_{-k}$ :

$\mathbf{N}^{w}=A M\left(\right.$ bid $_{\mathbf{k}}$, bid $\left._{-k}\right)$

Besides this, the strategy philosophy has to be expressed in a formal statement and measure in order to design algorithms. The aim of the bidding strategy is to maximize the utility difference of the utility won and the actual utility wanted under condition of a certain bid constellation for the critical data:

$\mathbf{S}_{3}=\left\{\mathbf{b i d} \mid \arg \max _{\text {bid }} \Delta_{\eta}\left(\mathbf{N}^{w, c r i}, \mathbf{N}^{b, c r i}\right)\right\}$

The set $\mathbf{S}_{3}$ includes all bids bid which maximize the utility difference function for the critical data. The same procedure will be applied for the uncritical data with respect to $\mathbf{S}_{3}$ :

$\mathbf{S}_{2}=\left\{\mathbf{b i d} \mid \arg \max _{\mathbf{b i d} \in \mathbf{S}_{3}} \Delta_{\eta}\left(\mathbf{N}^{w, u c r i}, \mathbf{N}^{b, u c r i}\right)\right\}$

Up to now, this set of bids maximize the utility difference of the uncritical data subject to the maximization of the utility difference of the critical data. After having calculated the set $\mathbf{S}_{2}$, the bid vector bid $\in \mathbf{S}_{2}$ will be chosen which minimizes the costs:

$\operatorname{bid}_{k} \in \mathbf{S}_{1}=\left\{\operatorname{bid} \mid \arg \min _{\mathbf{b i d} \in \mathbf{S}_{2}} c\right\}$

Especially, in a discriminatory auction the $\cos t c_{d i s}$ depending on the $\mathbf{b i d}_{i}$ and the number of GRECs $N_{l, i}^{b, y}$ can be expressed by:

$c_{d i s}=\sum N_{l, i}^{w, x, y} b_{i}$ 


\subsection{Strategy for incomplete information}

The bidding vectors which are intended to be submitted have to be covered by the bidding strategy not assuming a cooperation among the agent. Thus, the bidding vectors bid $_{-k}$ are unknown information resulting in using stochastic methods and the method only approaches the ideal bidding strategy. The number of goods won are no longer deterministic. The bid $_{-k}$ are characterized by a probability density $h\left(b_{-k}\right)$. The conditional probability $P\left\{N^{w} \mid \mathbf{b i d}_{k}\right\}$ of the goods won $N^{w}$ is subject to the own bid vector bid $_{k}$.

\subsection{Integral part-I}

The bidding strategy approaches the ideal strategy, by maximizing the expectation of the utility difference subject to the bid. Eqs. (8) and (9) are modified to:

$\mathbf{S}_{3}=\left\{\mathbf{b i d} \mid \arg \max _{\text {bid }} E\left\{\Delta_{\eta}\left(\mathbf{N}^{w, u c r i}, \mathbf{N}^{b, c r i}\right) \mid \mathbf{b i d}\right\}\right\}$

$\mathbf{S}_{2}=\left\{\mathbf{b i d} \mid \arg \max _{\mathbf{b i d} \in \mathbf{S}_{3}} E\left\{\Delta_{\eta}\left(\mathbf{N}^{w, u c r i}, \mathbf{N}^{b, u c r i}\right) \mid \mathbf{b i d}\right\}\right\}$.

The minimization of the cost is transformed into a worst case estimation resulting in the minimization of the maximum expected costs Eq. (10):

$\operatorname{bid}_{k} \in \mathbf{S}_{1}=\left\{\operatorname{bid} \mid \arg \min _{\mathbf{b i d} \in \mathbf{S}_{2}} \max c\right\}$

specifically for a discriminatory multi-unit sealed-bid auction:

$\max c_{d i s}=\sum N_{l, i}^{b, x, y} b_{i}$

The selection of the expectation instead of a probability optimization is based on the right balance of a priori calculated losses and the probability that this utility will occur. For instance, choosing a maximum a posteriori or a maximum likelihood estimator, both maximizes probabilities of either the value of the utility difference subject to the bids or vice versa. Consequently, in latter cases the measure of both the utility and the corresponding occurring probability is missed.

\subsection{Histogram representation}

Based on the histogram $\tilde{h}\left(x \mid r, N_{d e m}\right.$, won $)$ which collects the bids won under the additional conditions reserve price $r$ and number of bids won $N_{d e m}$ reflecting the influence of the demand, the expected GRECs won under a certain bid vector constellation will be determined. Clearly, the histogram mainly changes its shape by choosing a different $r$ because of the fixed maximum users' purchase power and by getting a demand higher equal than or less than the number of GRECs $N_{\max }$ offered. If $N_{d e m} \geq N_{\max }$ in the past auction, the histogram graph normally possesses a shape between $r$ and the maximum purchase power. Whereas, if $N_{d e m}<N_{\max }$, the bids will be concentrate close to $r$.

The histogram can be stored efficiently in a $2^{N_{b i t}+1} \times$ $2^{N_{b i t}+1}$ matrix (see Fig. 2). The first $2^{N_{b i t}}$ rows are reserved for $N_{\text {dem }}<N_{\max }$ and the second $2^{N_{b i t}}$ for the complementary statement. Each row of the two submatrices is devoted to a quantized reserve price in ascending order. Choosing a specific row for a proper reserve price and demand/supply ratio, the elements within the row are the number of the occurrence of the proper quantized bid. The bids are ordered equal to the matrix indices. In the last column the cumulative sums of the rest elements of the proper rows are stored in order to compute the relative frequency. The separation is done to keep the storage small and contemporarily provide enough accuracy for the proposed optimization algorithm.

\subsection{GRECs estimation}

Assuming that the bids bid $_{-k}$ of the other users are independent and identically distributed according to the relative frequencies of the histogram $\tilde{h}_{X}\left(x \mid r, N_{\text {dem }}\right)$, the probability $P\left\{x<b_{i}\right\}=H_{\kappa}^{k_{0}}\left(b_{i}\right)$, that given $k_{0}$ other bids at most $\kappa$ bids are higher than $\operatorname{bid}_{i}$, can be calculated:

$H_{\kappa}^{k_{0}}\left(b_{i}\right)=\sum_{l=0}^{\kappa-1}\left(\begin{array}{c}k_{0} \\ l\end{array}\right)\left(H_{X}\left(b_{i}\right)\right)^{k_{0}-l} \cdot\left(1-H_{X}\left(b_{i}\right)\right)^{l}$,

whereas $\kappa=\min \left\{N_{\max }, N_{d e m}\right\}$. If $\kappa>k_{0}$, Eq. (16) converges to a binomial progression and thus $H_{\kappa}^{k_{0}}\left(b_{i}\right)=1$. That is, if the demand $N_{\text {dem }}$ is smaller than the supply, the user will get $\kappa-k_{0}-1$ GRECs with probability 1. The corresponding probability density $h_{\kappa}^{k_{0}}\left(b_{i}\right)$ to Eq. (16) which will be used in the proposed algorithm is:

$$
\begin{aligned}
h_{\kappa}^{k_{0}}\left(b_{i}\right)= & k_{0} h_{X}\left(b_{i}\right)\left(\begin{array}{c}
k_{0}-1 \\
\kappa-1
\end{array}\right) \\
& \left(H_{X}\left(b_{i}\right)\right)^{k_{0}-\kappa}\left(1-H_{X}\left(b_{i}\right)\right)^{\kappa-1}
\end{aligned}
$$


The expected number of goods $N^{e}$ won given a proper bid constellation bid can be determined applying Eq. (16):

$$
\begin{aligned}
N^{e} & =E\left\{N^{w} \mid \mathbf{b i d}\right\}=\sum_{i=1}^{2 L} N_{l, i}^{e, x, y} \\
N_{l, i}^{e, x, y} & =\sum_{j=s N_{l-1, i}^{y}}^{s N_{l, i}^{b, x, y}} H_{N_{\max }-j}^{k_{0}}\left(b_{i}\right) \\
s N_{l}^{b, x, y} & =\sum_{u<l} N_{u}^{b, y}
\end{aligned}
$$

The GRECs estimated and the according bids are comprised in a tuple $\left(N_{l, i}^{e, x, y}, b_{i}\right)$.

\subsection{Derivation of the algorithm}

In the following it is assumed that the DATA-GREC mapping works optimally. That is for a given number of goods won $N^{w}$ the division to the link, QoS classes and categories maximizes the utility. Moreover the bids of the others are assumed to be independent and identically distributed. The own bids underly a differential budget constraint:

$0 \leq b_{i} \leq c_{i}$

The goal of the algorithm is to compute the solution of the strategy with incomplete information Eqs. (12)(14). The expectation of the utility difference subject to bid vector bid $_{k}$ can be written using order statistics:

$$
\begin{aligned}
E\left\{\Delta_{\eta} \mid \mathbf{b i d}_{k}\right\}= & \sum_{n=0}^{N_{\max }} E\left\{\Delta_{\eta} \mid N^{w}=n\right\} \\
& \cdot\left(H_{N_{\max }-n}^{N_{\max }}\left(b_{i_{n}}\right)-H_{N_{\max }-n+1}^{N_{\max }}\left(b_{i_{(n-1)}}\right)\right) \\
= & \sum_{n=1}^{N_{\max }} \sum_{z} \eta\left(\alpha_{z}, N_{z}^{w}, N_{z}^{b}\right) \\
& \cdot\left(H_{N_{\max }-n}^{N_{\max }}\left(b_{i_{n}}\right)-H_{N_{\max }-n+1}^{N_{\max }}\left(b_{i_{(n-1)}}\right)\right) \\
& -\sum_{z} \eta\left(\alpha_{z}, N_{z}^{b}, N_{z}^{b}\right) \\
\sum_{z} N_{z}^{w}= & N^{w}
\end{aligned}
$$

The maximization of Eq. (22) is equivalent to maximize the first sum in Eq. (23) with respect to the bid vector. Thanks to the conditional expectation notation, only the order statistics terms are depended on the bid vector. The cumulative probability function $H_{y}^{N_{\max }}(x<w)$ is both monotonically increasing in $y$ and $w$, therefore all summands are positive keeping in mind the nonnegative utility difference $\eta$. Taking now the assumption of the optimal allocation and that a bidding strategy bids at most for $N^{b}$ RRGs in the whole, the difference of two adjacent utility functions is always nonnegative:

$\left.\sum_{z} \eta\left(\alpha_{z}, N_{z}^{w}, N_{z}^{b}\right)\right|_{N^{b}}-\sum_{z} \eta\left(\alpha_{z}, N_{z}^{w}, N_{z}^{b}\right)_{N^{b}-1} \geq 0$

$\sum_{z} N_{z}^{b}=N^{b}$

Reordering Eq. (23) with respect to the cumulative distribution function gives a sum with nonnegative summands each of it increases in the bid value $b_{i_{n}}$ :

$$
\begin{aligned}
E\left\{\Delta_{\eta} \mid \mathbf{b i d}_{k}\right\}= & \sum_{n=1}^{N_{\max }} \sum_{z}\left(\left.\eta\left(\alpha_{z}, N_{z}^{w}, N_{z}^{b}\right)\right|_{N^{w}=n}\right. \\
& \left.-\left.\eta\left(\alpha_{z}, N_{z}^{w}, N_{z}^{b}\right)\right|_{N^{w}=n-1}\right) \cdot H_{N_{m} a x-n}^{N_{m} a x}\left(b_{i_{n}}\right) \\
& -\sum_{z} \eta\left(\alpha_{z}, N_{z}^{b}, N_{z}^{b}\right)
\end{aligned}
$$

Thus, the maxima of Eq. (26) can be found by only considering the probability density function (17). This equation is zero if the single density function $h_{X}\left(b_{i}\right)$ is zero or the according cumulative distribution function is 0 or 1 . The latter two cases are included in the first one. The global maximum of Eq. 26 for $b_{i_{n}} \in\left[0, c_{i_{n}}\right]$ is $c_{i_{n}}$, if $h_{n}^{N_{\max }}\left(c_{i_{n}}\right)$ is unequal zero. Otherwise there exists one single interval, in which the cumulative distribution function is globally maximum. To simplify matters, the kernel equivalence relation $\pi_{f}$ of a function $f(x), x \in$ $[a, b]$ is introduced:

$(x, y) \in \pi_{f} \Leftrightarrow f(x)=f(y)$

For this equivalence relation the equivalence classes $[x]_{\pi_{f}}$ are one-to-one.

\subsection{Algorithm}

The maximum costs $c_{i_{n}}$ are descending ordered. That is the definition interval of bid $b_{i_{(n+1)}}$ is a subset of the interval of $b_{i_{n}}$. The maximum reached by $b_{i_{n}}$ is at least as high than the one gained by $b_{i_{(n+1)}}$. Thus, bids are also descending ordered and this order relation are equivalent to the $c_{i_{n}}$ order relation. Therefore, the expectation of the utility difference $E\left\{\Delta_{\eta}\right\}$ is maximized by choosing the bids $b_{i_{n}}$ of $\left[c_{i_{n}}\right]_{\pi_{H_{X}}}$ and from this selecting 


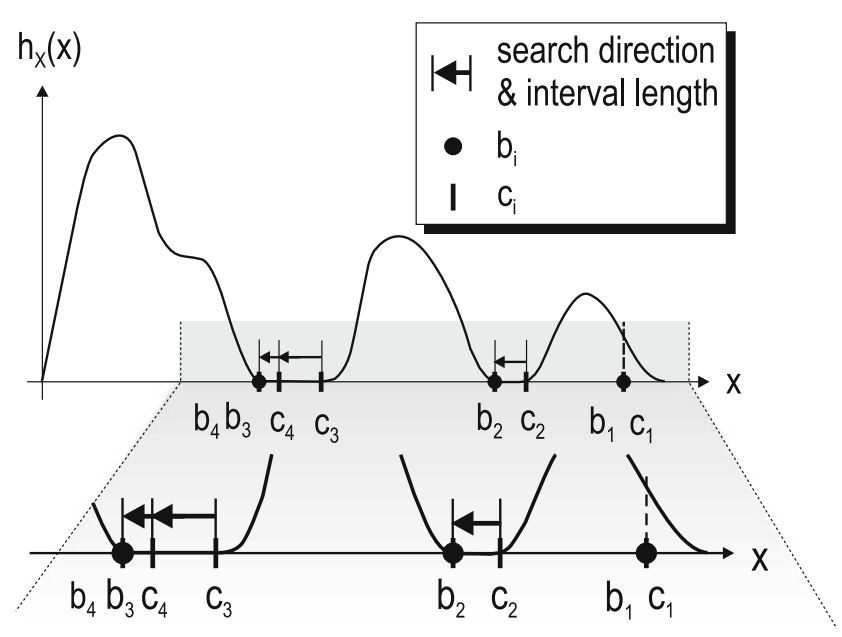

Figure 3 Algorithm concept

the minimum bid to minimize the maximum expected costs. Consequently the algorithm starts for the least significant bid $b_{i_{N_{\max }}}$ and steps down from $c_{i_{N_{\max }}}$ to the reserve price until $h_{X}$ is unequal zero. That is, given $h_{X}(x)$ with $x \in\left[r, c_{i_{N_{\max }}}\right]$, the first step can be expressed formally as:

$b_{i_{\max }}=\min \left[c_{i_{N_{\max }}}\right]_{\pi_{H_{X}}}$

For the second least significant bid the procedure is the same, but the searching space is at least reduced to

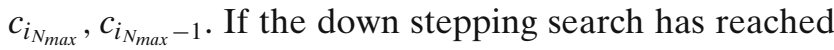
$c_{i_{N_{\max }}}$ without finding $h_{X}$ is unequal to zero, $b_{i_{N_{\max }-1}}$ is automatically equal to $b_{i_{N_{\max }}}$ :

$b_{i_{N_{\max }-1}}=\min _{x}\left\{\left[c_{i_{N_{\max -1}}}\right]_{\pi_{H_{X}}}\right\}-\left\{x \mid x<b_{i-\left(N_{\max }+1\right)}\right\}$

The following steps are similar to the second one in finding the corresponding bids. The searching space for the $k^{\text {th }}$ bid $b_{i_{k}}$ is at least to the last maximum cost limit $c_{i_{k+1}}$, hence Eq. (29) can be generalized to:

$b_{i_{k}}=\min _{x}\left\{\left[c_{i_{k}}\right]_{\pi_{H_{X}}}\right\}-\left\{x \mid x<b_{i_{(k+1)}}\right\}$

Figure 3 shows an example for the algorithm procedure. It is assumed that there are four cost constraints $c_{i}$ which are sorted in descending order. The algorithm starts with the lowest cost constraint $c_{4}$ and searches in the negative abscissa direction until the probability density $h_{X}(x)$ is unequal zero. This abscissa value is $b_{4}$. Thereafter the search starts from $c_{3}$. The complete interval $\left[c_{4}, c_{3}\right]$ does not possess an $h_{X}(x)$ value unequal zero, thus $b_{3}$ is set to $b_{4}$. For $b_{2}$, the same procedure as for $b_{4}$ occurs. The search for a non-zero $h_{X}(x)$ within $x \in\left[c_{2}, c_{1}\right]$ stops immediately, because of $h_{X}\left(c_{4}\right)$ being unequal to zero.

\subsection{Computational effort}

The computation time of the algorithm is independent of the number of users and goods, but at most linear to the number of quantization steps of the bids assuming bids with the same maximum costs are bundled. This can be seen, because this algorithm scans partly distinguished intervals of the bid space and searches there for maxima of $H_{X}$.

\subsection{Differential part-II}

The bid vector which is chosen out of the set $\mathbf{S}_{1}$ is the result of the integral part taking long history into account. If the main auction parameter $r$ and $N_{d e m}$ remain stable and thus the same history information will be used, the differential part is activated in order to decrease or increase the bid by \pm bidunit. The algorithm can be roughly described in two cases:

- If $N_{l}^{w, y}=N_{l}^{b, y}$ in the past auction $\wedge \exists b_{-k}<b_{i}$ in $\Rightarrow$ $b_{i}=b_{i}-$ bidunit

- If $N_{l}^{w, y}<N_{l}^{b, y}$ in $a_{t-T} \Rightarrow b_{i}=b_{i}+$ bidunit

The first case reduces bids if there are lower bids of the other users. On the other hand in the second case, if the user gets less GRECs than expected, the differential part increases the bids in order to improve the allocation. Additional constraints like $\mathbf{c}_{l}^{y}$ etc. are included in the algorithm.

\subsection{Output}

The bid vector bid will be sent to the auctioning mech-

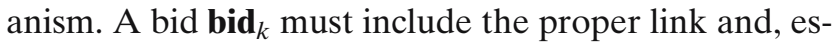
pecially for the downlink, the proper QoS-class must be highlighted. This information is needed afterwards to allocate the resource. If this information is missing, the auction mechanism is not able to allocate the resources concerning technical constraint.

\section{Reserve price calculator}

Besides the BIS a UT can influence the auction process, the RPC is the operator's leverage. The RPC aims at maximizing the Operator's monetary gain. Its functionalities must be adapted to the proper AM of the specific RAT. Generally, the RPC gets information of future events to determine $r$, but there are also information of the past bidding behaviors expressed by bid $_{k}$ and the past auction conditions. 
The RPC functionalities can possess both a differential and an integral part which is described in the following.

The memory of the RPC is a vector RPC whose components are triples of the form $\left(r_{j}, g_{j}, t_{j}\right)$. The number of components is equal to the number of reserve price steps. Each component incorporates the gain $g_{j}$ which was reached $t_{j}$ auction periods ago by an announced reserve price $r_{j}$. The entry $\mathbf{R} \mathbf{P C}{ }_{j}$ will be modified, either if the auctioneer currently proclaims $r_{j}$ or if the maximum memory time to life is reached, that is $t_{j}=T_{R P C}$. In the first case, the gain $g_{j}$ is set to the actual gain of the auction and $t_{j}=0$. Otherwise, the gain $g_{j}$ is set to -1 which indicates no value available. If these cases do not occur, all timers counter of the other components $\mathbf{R P C}_{-j}$ will be increase by $T$.

The RPC determines the reserve price regarding the RPC, the gain $g(t-T)$ reached in the past auction, the current gain $g(t)$ and the Worse Timer (WT).

The function can be distinguished in two decisions at first glance:

First, if $g(t)>g(t-T)$, that is if the gain increases, the reserve price won't be changed and the WT is set to zero. Second, if $g(t-T) \geq g(t)$, three cases are possible:

1) If the nearest upper and lower $r_{j}$ neighbor $r_{j+}$ and $r_{j-}$ exist and their proper gains $g_{j+}$ and $g_{j-}$ are smaller than $g_{j}$, then $r_{j}$ won't immediately be changed, but the WT will be decreased by 1 . If the WT is zero, based on the decreasing tendency, the reserve price is changed by one step in direction of the higher derivation $\frac{\partial g}{\partial r}$, afterwards the WT is set to zero.

2) If both $r_{j+}$ and $r_{j-}$ exist and one's gain is higher, the other is smaller than $r_{j}$, the reserve price will instantaneously be changed in direction of the derivation and the WT is set to zero.

3) If neither $r_{j+}$ or $r_{j-}$ exists, the reserve price will be changed in direction of the derivative and the WT is set to zero.

After this calculation the reserve price $r(t)$ and $N_{\max }$ will be sent to the auction participants.

A reserve price will be considered as the border of the definition set for a duration $T_{R P C}$, if it was taken, but the sum of $N^{w}$ was zero. RPC takes the history into account by RPC . This history is used to determine the derivation $\frac{\partial g}{\partial r}$ being responsible for a change of the reserve price. This differential part will again be stabilized by the delay of WT. All this functions need less buffer and computation power what was one constraint of the RPC design.

\section{Auction mechanism}

The Auction Mechanism aims at allocating the GRECs according to a proper auction method and with respect of the technical constraints given by the RAT. In the following it is always referred to a Discriminatory Multi-unit Sealed-bid Auction (DMSA), if not stated otherwise. The main characteristic of the DMSA is, that the bidders have to pay their bids for the goods won.

This system can dynamically allocate the UL and DL resources within an auction period. Assuming that each GREC can be individually taken for either UL or DL regardless of the other GRECs, the DSMA is optimal and efficient according the corresponding terms in auction theory, if the highest bids win the goods. Otherwise, if this is not fulfilled based on technical constraints, modified allocation mechanisms are needed in order to optimize the operator's gain.

1) Input: The RPC conveys $r$ and the UTs transmits their bids. The bids are mapped to a set of 5-tuple

$$
A=\left\{\left(N_{l, i}^{b, x, y}, b_{i}, y, l, m\right) \mid m=U T-I D\right\}
$$

whose four first components are the bid $_{k}$ of the user possessing the User Terminal IDentification UT$I D=m$.

2) Allocation: Assuming independent GRECs, the AM sorts the elements of $A$ with respect to $b_{i}>$ $r$ in a decreasing manner. The highest bids win GRECs while the sum of GRECs won is at most $N_{\max }$.

3) Output: The AM informs all participants of the outcome by broadcasting the allocation vector $x$ ${ }_{j}\left(N_{l, j}^{w, x, y}, b_{j}, y_{j}, l_{j}, m_{j}\right)$. This information is the input to the BIS, the MAC scheduler and the PHY in UL/DL.

\section{Simulations}

The environment simulated is one cell. A BS controlls the RRGs of proper numbers of UTs, whereas the EM manages the GRECs. The BIS gets the critical number of GRECs $N_{l}^{q, y}$ which should be sent within this auction period to fulfill the QoS and the uncritical data as an input from the DGM. The DGM divides the data optimally into the RRGs. The traffic is assumed to have a constant data rate which needs for service class $S C_{1}$ 3.5 GRECs and for $S C_{2} 3$ GRECs per auction. The same simulation was done by replacing the constant data rate with a Poisson arrival process of the data and an average data rate of both SCs as the same as 
for the constant data rate. For the sake of simplicity the DL is not considered. This results in a $65 \%$ GREC need per user. That is, this cell is designed to transport the critical data of approximately two users. The QoS is characterized by a maximum delay for the QoS. To fulfill the QoS the data has to transmit within 1.5 times the auction period duration after arriving in the $S C$ buffer.

Both the preferences parameter $\alpha_{l}^{y}$ and the max. costs $\mathbf{c}_{l}^{y}$ are independently distributed parameters in $[0,1]$. It is assumed that the bidders choose for all QoS classes a risk-neutral behavior, i.e.,

$\eta_{l}^{x, y}\left(\alpha_{l}^{x, y}, N\right)=\alpha_{l}^{x, y} N$.

Furthermore, the RPC calculates the reserve price $r$ according to the algorithm described in Section 5. These inputs of the BIS lead to bidding vectors which are sent to the DSMA.

To measure the performance of the bidding strategy the marginal QoS $\mu_{l}^{x, y}$ is considered. The marginal QoS is the quotient between the data sent and the critical data in the buffer, what can be expressed by the number of GRECs:

$\mu_{l}^{x, y}=\frac{N_{l}^{w, x, y}}{N_{l}^{b, x, y}}$.

The proposed bidding algorithm is compared with the ideal bidding strategy in terms of maximum QoS and maximum utility difference. Figure 4 shows the marginal QoS of $S C_{1}$ and $S C_{2}$ depending on the number of users. The $S C_{1}$ possesses higher preferences than $S C_{2}$, therefore the bidding strategy cares more about $S C_{1}$ and thus the $Q o S_{1}$ fulfillment is better than for $Q o S_{2}$. The dashed lines indicates the performance of

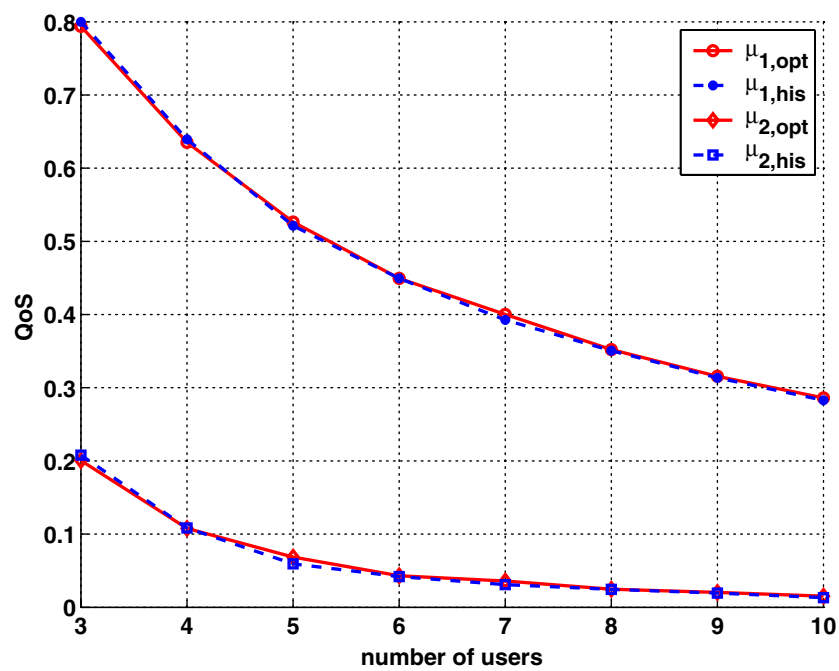

Figure 4 Marginal QoS $\mu_{l}$ depending on the number of users $x$

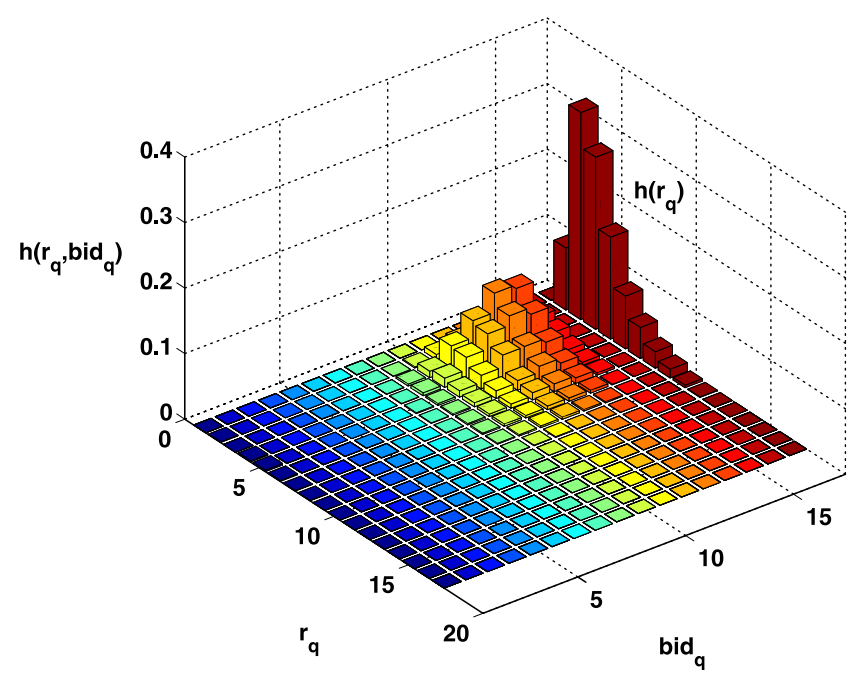

Figure 5 Histograms $h\left(r_{q}\right)$ and $h\left(r_{q}, b_{q}\right)$ depending of the quantized reserve price $r_{q}$ and bid $b_{q}$

the ideal bidding strategy. The proposed bidding strategy possesses approximately the same performance. The performance for the constant traffic and the bursty traffic is the same. The QoS decreases if the number of users increases, because the demand increases.

To get a feeling about the dynamic, Fig. 5 shows the relative frequency $h\left(r_{q}, b_{q}\right)$ of the quantized reserve price $r_{q}$ and the bids $b_{q}$ for the case of four users, $N_{b i t}=$ 4 and $N_{\text {dem }} \geq N_{\text {max }}$. This relative frequency is the upper submatrix in Fig. 2. The numbers of quantization steps for $r$ and $b$ are the same. The reserve price is the minimum reserve price $r_{\min }$ for $r_{q}=0$ and oppositely the maximum reserve price is reached by $r_{q}=15$. That is, the higher $r_{q}$ is, the less is the probability that the bids will exceed it as shown in Fig. 5. Furthermore, the higher $r_{q}$ is, the smaller the absolute bid interval will be. Recalling from Section 3.2.1, the absolute bid value for the same $b_{q}$, but a higher $r_{q}$, is higher. That is, for absolute value consideration the relative frequency in Fig. 5 has to be compressed an shifted towards higher bids. Resulting in higher and less bids as mentioned above.

In the back of the Fig. 5 at $b_{q}=16$ the relative frequency $h\left(r_{q}\right)$ of $r_{q}$ is shown separately. The relative frequency $h\left(r_{q}, b_{q}\right)$ decreases to lower bids because the demand is higher than the supply. Otherwise, the relative frequency is concentrated at $r_{q}=0$. Furthermore, the RPC tries to increase the reserve price as high as possible to increase the bids, but simultaneously the number of bids which can exceed the reserve price decrease and thus the monetary gain decreases. The controlling of this trade-off results in a high relative frequency $h\left(r_{q}\right)$ for lower $r_{q}$. 


\section{Conclusion}

The proposed allocation and pricing mechanism aims at terminals which have Cognitive Radio abilities, that is which learn about the environment and act accordingly to optimize its position in a dynamic environment. The multi-unit sealed-bid auctions provides a proper allocation mechanism which can be implemented in algorithms within the MAC to execute fast repeated auctions. Therefore, the RAT can fast adapt to market in a very much local area like a cell. Intelligent algorithms take care to observe the environment and act to optimize the utility of the proper person represented. The proposed bidding algorithm based on order statistics is independent in the number of RRGs and users and linear in the number of quantization steps. This algorithm possesses a similar performance as the ideal bidding strategy in terms of QoS fulfillment and utility difference maximum.

Acknowledgements This work is partially funded by the Commission of the European Communities, under the 6th Framework Program for Research and Technological Development, within the project End-to-End Reconfigurability $\left(E^{2} R\right)$. The authors would like to acknowledge the contributions of their colleagues from $\mathrm{E}^{2} \mathrm{R}$ consortium.

\section{References}

1. Mitola III J (1999) Cognitive radio for flexible mobile multimedia communications. In: IEEE international workshop, mobile multimedia communications, San Diego, California, 3-10 1999

2. Tanenbaum AS (1981) Computer network. Prentice-Hall, Englewood Cliffs, New Jersey

3. Bayer DL, Nutt GJ (1982, January) Performance of csma/cd networks under combined voice and data loads. IEEE Trans Commun 30(1):6-11

4. Colvin A (1983) Csma with collision avoidance. Comput Commun 6:227-235

5. Greenstein LJ, Amitay N (1994, November) Resource auction multiple access (rama) in the cellular environment. IEEE Trans Veh Technol 43(4):1101-1111

6. Nanda S, Amitay N (1994, August) Resource auction multiple access (rama) for statistical multiplexing of speech in wireless pcs. IEEE Trans Veh Technol 43(3):584-596

7. Amitay N (1992) Resource auction multiple access (rama): efficient method for fast resource assignment in decentralised wireless pcs. Electron Lett 28(8):799-801

8. Roberto Boisson de Marca J, Javier Santivanez G (1997) D-rama: A new deterministic mac protocol for wireless multimedia communications. In: The 8th IEEE international symposium on personal, indoor and mobile radio communications, Helsinki, Finland, 1043-1048 1997

9. Krishna V (2000) Auction theory. Academic, San Diego, California

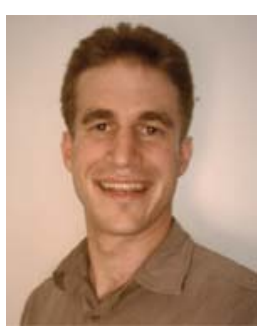

Clemens Kloeck (S'03) received the Dipl.Ing. degree in electrical engineering from the Universitaet Karslruhe (TH) in 2002 and is currently working towards his Ph.D. degree in dynamic and distributed wireless communication systems at the Universitaet Karlsruhe (TH). He works within the End-to-End Reconfigurability (E 2 R) project in direction of Cognitive Radio, Cognitive Networks and dynamic spectrum sharing.

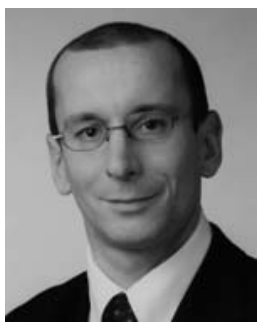

Holger Jaekel received a Diploma degree in applied mathematics (Dipl.-Math. techn.) in 1999 and a Doctoral degree in Electronic Engineering and Information Technology (Dr.-Ing.) in 2003, both from the Universitaet Karlsruhe (TH). From 1999 to 2003 he worked as a research associate. Since 2003 he has been a senior researcher within the Communications Engineering Laboratory. His current research interests are in ultra wideband communications, mobile communications, signal processing in communications and dynamic spectrum allocation. Currently Dr. Jaekel is involved in several projects, focussed on but not restricted to ultra wideband communications.

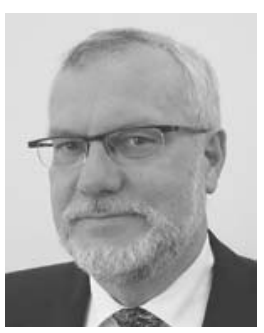

Friedrich K. Jondral (SM'94) received Dipl.-Math. and Dr. rer. nat. degrees in mathematics from the Technical University of Braunschweig (Germany), in 1975 and 1979, respectively. From 1979 to 1992 he was with AEG-Telefunken (now European Aeronautic Defence and Space Company, EADS), Ulm (Germany), where he held various research, development, and management positions. During this time he also lectured on courses in applied mathematics at the University of Ulm, where he was appointed Professor in 1991. Since 1993 he has been Full Professor and Head of the Communications Engineering Lab at the Universitaet Karlsruhe (Germany). His current research interests are in the fields of mobile communication, spread spectrum, signal analysis, pattern recognition, network capacity optimization and dynamic channel allocation. 